\title{
A Pediatric Case of Atypical Chronic Myeloid Leukemia with CSF3R Mutation Not Responding to Ruxolitinib, but Rescued by Allogeneic Transplantation
}

\author{
Yoon Jung Choi ${ }^{1}$, Hee Jo Baek ${ }^{1,3}$, Bo Ram Kim¹, Soo Min Park', Myung-Geun Shin ${ }^{2}$ and Hoon Kook ${ }^{1,3}$ \\ Departments of ${ }^{1}$ Pediatrics and ${ }^{2}$ Laboratory Medicine, Chonnam National University Hwasun Hospital, Hwasun, \\ ${ }^{3}$ Department of Pediatrics, Chonnam National University Medical School, Gwangju, Korea
}

\begin{abstract}
Atypical chronic myeloid leukemia (aCML), BCR-ABL1-negative is a rare myeloid neoplasm, especially in pediatric patients. The mutations identified in aCML have overlapped with those of other myeloid neoplasms. In recent studies, ruxolitinib, a Janus kinase (JAK) inhibitor, was found efficient in some patients of aCML with CSF3R mutation. Here, we report a pediatric case of aCML with CSF3R mutation who did not respond to ruxoritinib, but was successfully rescued with hematopoietic stem cell transplantation (SCT). A stuporous 13-year-old boy was transferred with leukocytosis. Computed tomography showed an acute lobar intracranial hemorrhage in the left frontal lobe. The bone marrow aspirate demonstrated significant granulocytic proliferations with predominant dysplasia. Hydroxyurea and imatinib were initially administered to reduce leukocytosis. After $B C R-A B L 1$ was found to be negative, imatinib was discontinued. After the identification of CSF3R mutation by customized targeted DNA sequencing (NGeneBio, Seoul, South Korea), ruxolitinib was added. He seemed to have hematologic and clinical responses on 2 months of ruxolitinib treatment, but the blast counts in the bone marrow increased. He underwent a full-matched unrelated peripheral blood SCT successfully 3 months after his diagnosis and has currently been disease-free 8 months since the transplantation. In conclusion, ruxolitinib for aCML with CSF3R mutation might not always induce a significant response but could be used as bridge to hematopoietic SCT.
\end{abstract}

Key Words: Atypical chronic myeloid leukemia, CSF3R mutation, Ruxolitinib, Children
pISSN 2233-5250 / eISSN 2233-4580 https://doi.org/10.15264/cpho.2021.28.2.93 Clin Pediatr Hematol Oncol 2021;28:93 97

Received on September 28, 2021 Revised on October 6, 2021 Accepted on October 7, 2021
Corresponding Author: Hee Jo Baek
Department of Pediatrics,
Chonnam National University
Hwasun Hospital, Chonnam
National University Medical School,
322 Seoyang-ro, Hwasun-eup,
Hwasun 58128, Korea
Tel: +82-61-379-7695
Fax: +82-61-379-7697
E-mail: swan93@naver.com
ORCID ID: orcid.org/0000-0003-3830-8134

\section{Introduction}

Atypical chronic myeloid leukemia (aCML) is a rare $B C R-A B L 1$-negative myelodysplastic syndrome/myeloproliferative neoplasm (MDS/MPN). It is a disease of the elderly as the median age at diagnosis is in the sixth and seventh decades of life [1]. The incidence was estimated to be $1-2 \%$ of $B C R-A B L 1$-positive CML cases in adults, but rarer in children [2].

Currently, the diagnosis of aCML is based on morphol- ogy because its clinical and molecular features overlap with those of other myeloid neoplasms, such as chronic myelomonocytic leukemia, chronic neutrophilic leukemia (CNL), and unclassifiable MDS/MPN [1,3]. The main features of aCML are the principal involvement of neutrophil lineage ( $\geq 10 \%$ of the leukocyte) with leukocytosis

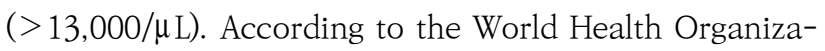
tion, 2016 diagnostic criteria, prominent granulocytic dysplasia, $<20 \%$ blasts in the peripheral blood (PB) and bone marrow (BM), and minimal absolute basophils $(<2 \%$ of leukocytes) and monocytes ( $<10 \%$ of leukocytes) 
should be entertained [4]. In addition, to rule out Philadelphia-positive CML and other myeloid-lymphoid neoplasms associated with eosinophilia, BCR-ABL1, PDGFRA, $P D G F R B$, and FGFR 1 rearrangements or PCM-Janus kinase (JAK)2 fusion must be excluded [4].

In contrast to $B C R-A B L 1$, which defines CML, no single genetic lesion characterizes aCML. The mutations identified in aCML are commonly found in other myeloid neoplasm cases $[1,3,5,6]$. The most commonly affected genes (>20\%) in aCML are SETBP1, ASXL1,N/K-RAS, SRSF2, and TET2, and less frequently ( $<10 \%) C B L, C S F R 3, J A K 2$, EZH2, ETNK1 [1,3,6]. Additionally, aCML has been reported to harbor JAK2 mutations [1,3]. Among them, CSF3R mutation can activate JAK/STAT signaling [3,5]. In recent studies, ruxolitinib, a JAK inhibitor, was found to be efficient in some patients with aCML with CSF3R mutation $[5,7,8]$.

Here, we report a pediatric case of aCML with CSF3R mutation who did not respond to ruxolitinib, but was successfully rescued by hematopoietic stem cell transplantation (HSCT).

\section{Case Report}

A 13-year-old boy in a stupor was admitted. He experienced a headache, vomiting two days ago, and weight loss of $5 \mathrm{~kg}$ (11.6\% of baseline weight) during the last 6 months. At the time of admission, his Glasgow coma scale score was 7 and his blood pressure was 164/78 mmHg. His left pupil was dilated and showed no reflex to the light. Physical examination revealed multiple bruises and spleen was palpable to $7 \mathrm{~cm}$ below the costal margin. The laboratory findings were as follows: white blood cells (WBC) of 296,500/ $\mu \mathrm{L}$, neutrophils of $177,900 / \mu \mathrm{L}$ (60\% of WBC), lymphocytes of $14,800 / \mu \mathrm{L}$ ( $5 \%$ of WBC), basophils of $3,560 / \mu \mathrm{L}$ (1.2\% of WBC), hemoglobin level of $7.4 \mathrm{~g} / \mathrm{dL}$, platelet counts of $27,000 / \mu \mathrm{L}$, prothrombin time of 15.8/55.7/1.38 secs/\%/INR, and activated partial thrombin time of 26.8 secs.

Brain computed tomography (CT) showed an acute lobar intracranial hemorrhage (ICH) in the left frontal lobe with the midline shifting to the right (Fig. 1A). After pla- telet transfusions, evacuation of the hematoma was attempted.

A PB smear showed left-shifted myeloid hyperplasia with dysplasia, blasts of $2 \%$, increased granulocytic precursors (myelocytes of 20\% and metamyelocytes of 3\%), and an absence of both basophilia and monocytosis (Fig. 1B). A BM aspiration revealed increased granulocytic series with $8.7 \%$ blasts (Fig. 1C). The blasts cells expressed CD13, CD33, CD34, CD56, CD117, and myeloperoxidase O. These led to a putative diagnosis of chronic CML. Hydroxyurea and imatinib were initially administered to reduce leukocytosis. On the third day of hospitalization (HD), fluorecence in situ hybridization (FISH) for $B C R$ $A B L 1$ was negative; thus, imatinib was discontinued.

BM cytogenetics was $46, \mathrm{XY}$, and the multiplex real-time polymerase chain reactions for 28 chromosome translocations, including $B C R-A B L$, were negative. Results of FISH for FIP1L1/PDGFRA, PDGFRB, and FGFR1 rearrangement were negative. Customized targeted DNA sequencing (NGeneBio, Seoul, South Korea) of 119 genes, including CALR, JAK2 or MPL, SETBP1, ETNK1, ASXL1, TET2, and $N / K R A S$ revealed mutations in CSF3R gene, c.1853C $>$ T (p.T618I) (variant allele frequencies, VAF 40.89\%) and c.1919C>A (p.T640N) (VAF 10.7\%). Also, PCM1-JAK2 fusion was not found. These results led to the diagnosis of aCML with CSF3R mutation.

After the initial aspiration of ICH, conservative measures using mannitol, dexamethasone, levetiracetam, and platelet transfusions were continued. On HD3, however, abrupt dilatation $(8 \mathrm{~mm})$ and loss of light reflex in the left pupil were noted, and a repeat brain CT revealed increased amount of ICH. Repeat aspiration of the hematoma resulted in decrease of ICH. The impaired patient's memory, orientation, and motor function of the right side gradually recovered over 2 weeks. The spleen was no longer palpable and leukocytosis and blast counts in the BM were normalized 4 weeks after the patient was started on hydroxyurea (Fig. 2).

On HD 28, upon the identification of CSF3R mutation, ruxolitinib was initiated at $35 \mathrm{mg} / \mathrm{m}^{2}$ per day due to thrombocytopenia, and hydroxyurea was discontinued. Ruxolitinib was used off label. As a result of decreased 

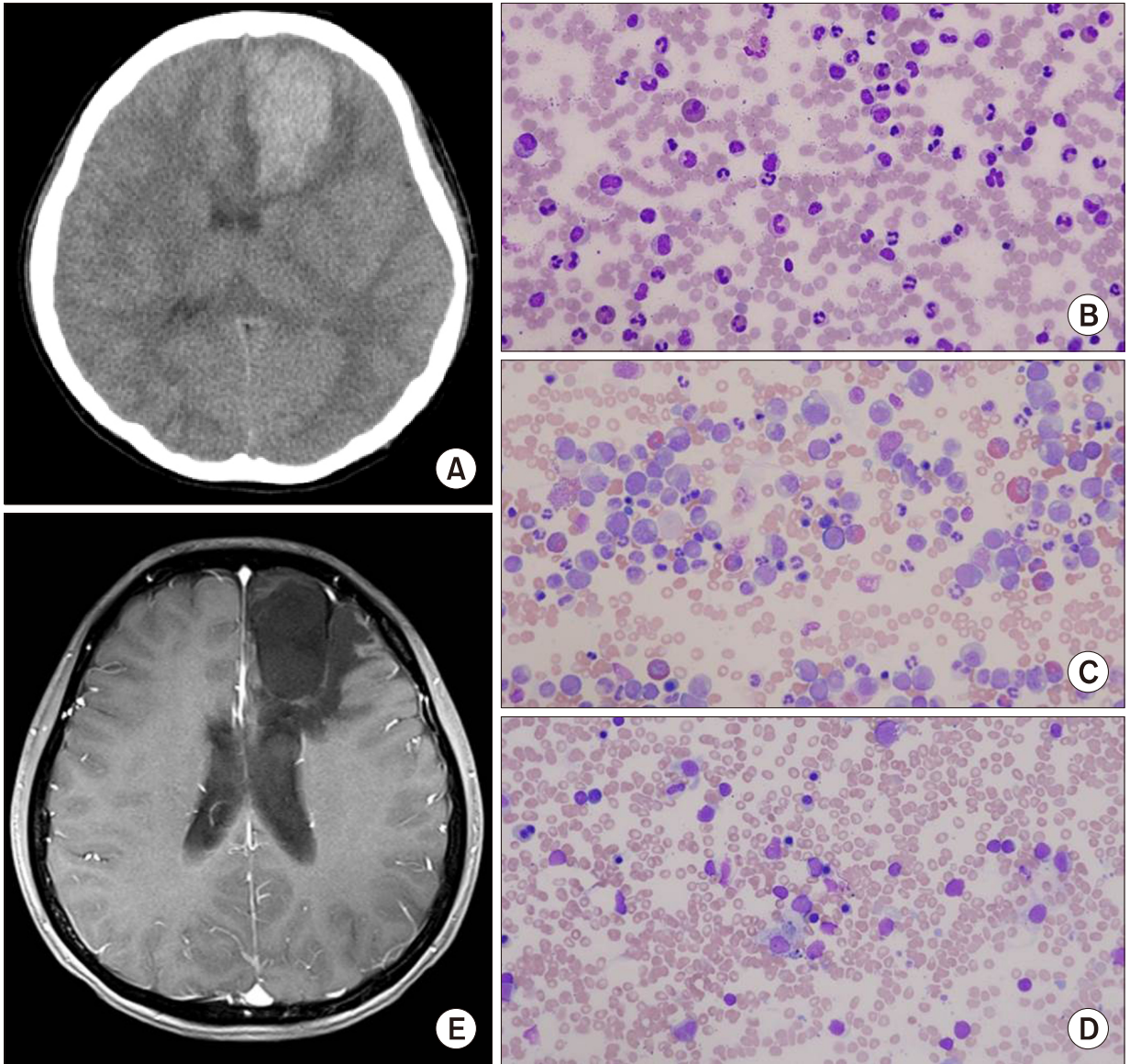

\section{.}
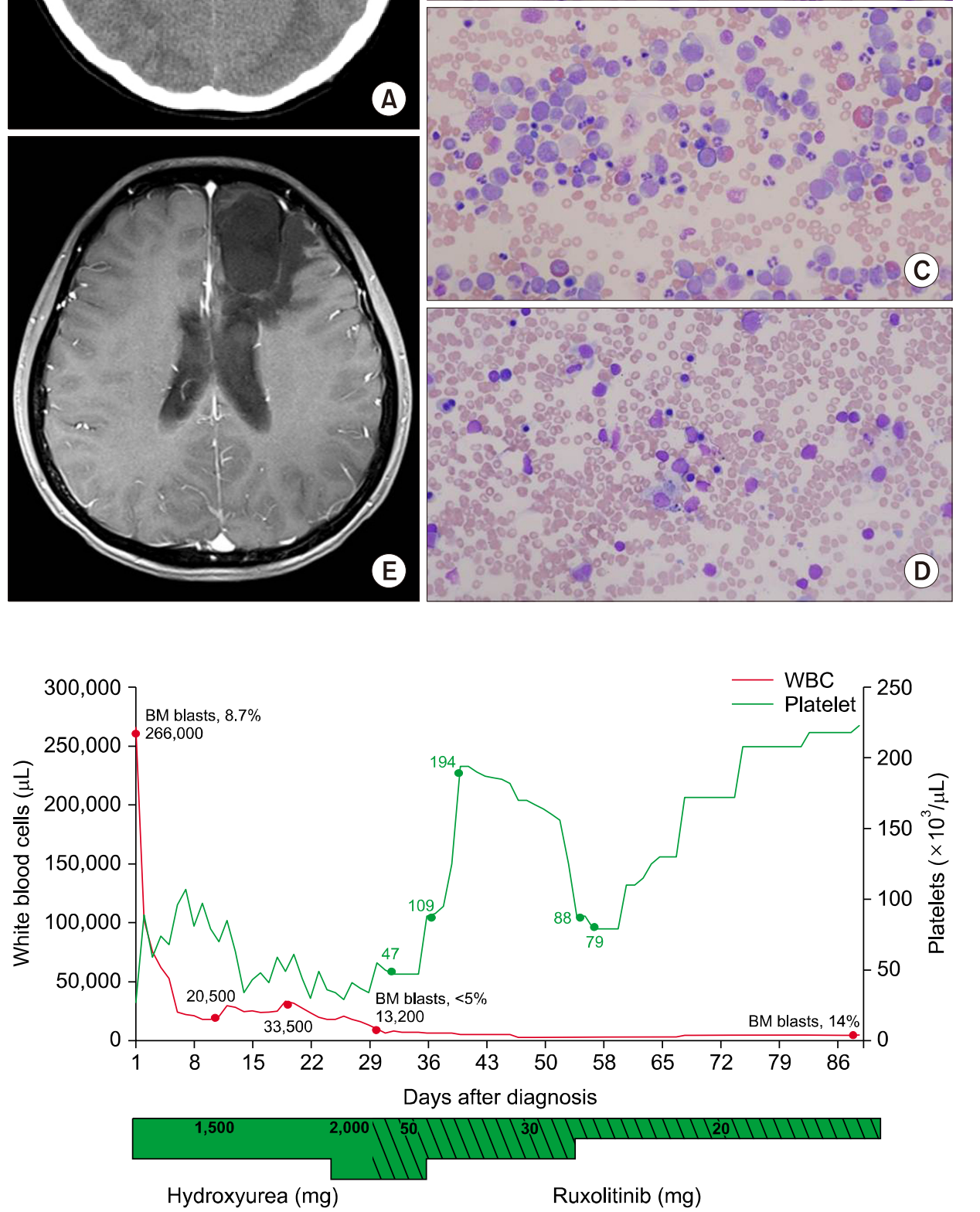

Fig. 1. (A) The computed tomography of the patient's brain at admission shows a $6.5 \mathrm{~cm}$ acute intracranial hemorrhage in the left frontal lobe with perilesional edema and midline shifting to the right. (B) An initial peripheral blood smear reveals left-shifted myeloid hyperplasia with dysplasia (myeloblasts $2 \%$, myelocytes $20 \%$ and metamyelocytes $3 \%$ of the leukocytes) (Weight-Giemsa stain, $\times 400$ ). (C) A bone marrow smear reveals increased granulocytic series with $8.7 \%$ myeloblasts and dysgranulopoiesis (Weight-Giemsa stain, $\times 400$ ). (D) A follow-up bone marrow smear of the patient 3 months after the initial evaluation shows increased myeloblasts of $14 \%$ (Weight-Giemsa stain, $\times 400$ ). (E) Magnetic resonance imaging of brain 11 months later shows encephalomalacic change with hemosiderin deposit in left frontal lobe and corpus callosum, sequelae of previous intracranial hemorrhage.

Fig. 2. Laboratory findings, including white blood cell counts, platelets in the peripheral blood, and blast counts in the bone marrow over 90 days from diagnosis to hematopoietic stem cell transplantation.
WBC and platelet counts, the ruxolitinib dosage was reduced to $13 \mathrm{mg} / \mathrm{m}^{2}$ per day and maintained for 10 weeks as a bridge to HSCT, according to a recent report [2]. However, at 2 months of ruxolitinib treatment, which was 3 months after the diagnosis, a follow-up BM aspiration revealed increased blasts of 14\% (Fig. 1D).

The patient received planned allogeneic peripheral blood stem cell transplantation from an $8 / 8$ allele- 
matched unrelated donor. The conditioning regimen consisted of fludarabine ( $40 \mathrm{mg} / \mathrm{m}^{2}$ for 5 days) and busulfan (3.2 mg/kg for 3 days). Graft versus host disease (GvHD) prophylaxis consisted of rabbit antithymocyte globulin $(1.25 \mathrm{mg} / \mathrm{kg}$ for 3 days), tacrolimus, and low doses of methotrexate. The time to neutrophil and platelet engraftment were $11^{\text {th }}$ day, $12^{\text {th }}$ day after transplant, respectively. Post-transplant 6 months, at one month after tacrolimus-off, he developed stage 3 gut GvHD which was treated with steroid and ruxolitinib.

Currently, his motor function has fully recovered, although magnetic resonance imaging of his brain showed encephalomalacic changes with hemosiderin deposit in the left frontal lobe and corpus callosum, sequelae of previous ICH (Fig. 1E). He remains in hematologic remission with complete donor chimerism at 8 months after transplant with manageable gut GvHD.

\section{Discussion}

Making the diagnosis of aCML in children is quite challenging, as they are extremely rare, and they may share clinical, morphologic and molecular similarities with other MDS/MPDs. Mutation in the CSF3R gene was found in $50-80 \%$ of cases of $\mathrm{CNL}$, but $<10 \%$ in aCML $[1,6]$. However, in this case, the morphology of the BM and $\mathrm{PB}$ revealed increased neutrophil precursors of $23 \%$ (>10\% of leukocytes), some segmented or banded neutrophils in $\mathrm{PB}$, and the presence of dysgranulopoiesis. These findings were compatible with those of aCML.

The natural history of aCML is characterized by an increasing leukemic cell burden, organomegaly, and a high rate of transformation to acute myeloid leukemia in $37-40 \%$ of aCML cases $[1,2,9,10]$. There is no current standard of care, but the only curative treatment option is allogeneic HSCT [1,2]. Traditionally, treatments used in MDS/MPN cases (such as hypomethylating agents, hydroxyurea, pegylated-interferon-alpha, and erythropoietic stimulating agents) have been utilized to control leukocytosis and symptomatic splenomegaly [2]. Recently, owing to the discovery of mutations in aCML, promising targeted therapies were investigated, including the JAK in- hibitor ruxolitinib, SRC kinase inhibitor dasatinib, and MEK inhibitor trametinib [1,11].

CSF3R mutation has two types [1,6]. First, membrane proximal mutations, such as CSF3R-T618I and CSF3R$T 640 N$ activate the JAK/STAT pathway and are inhibited by ruxolitinib. Second, truncation mutation activates SRC family-TNK2 kinase signaling and is sensitive to SRC kinase inhibitors, such as dasatinib. A few cases have reported encouraging results after treatment with ruxolitinib in aCML with CSF3R mutation [7,12,8]. Among these reports, there was only one pediatric patient who showed a robust response to ruxolitinib and then successfully underwent a HSCT [7]. Recently, in a phase II study of ruxolitinib with 44 adult patients (21 CNL and 23 aCML), irrespective of CSF3R mutation status, 35\% of the patients had an overall response (complete and partial response), and among them, 50\% had oncogenic CSF3R mutations. In $16 \mathrm{CNL}$ and $6 \mathrm{aCML}$ cases with CSF3R mutation, 13 and 1 patients had responses, respectively [5]. A Children's Oncology Group Phase I study for relapsed or refractory solid tumors, MPN, and leukemia recommended the ruxolitinib dose of $50 \mathrm{mg} / \mathrm{m}^{2} /$ dose twice a day [13].

In this case, after the identification of CSF3R T618I and CSF3R T640N mutations, ruxolitinib was initiated at $35 \mathrm{mg} / \mathrm{m}^{2}$ per day due to low blood counts. Unfortunately, after treatment of ruxolitinib for 2 months, his BM blast counts were increased, even though he maintained clinical and hematologic responses.

To date, the largest study of allogeneic HSCT for aCML has been reported from the European Society for Blood and Marrow Transplantation registry [14]. In 42 adult patients with aCML between 1997 and 2006, the 5-years relapse-free survival following HSCT was 36\%, and non-relapse mortality was $24 \%$, while relapse occurred in $40 \%$. Their median time from diagnosis to transplant was 7 months. There are no current recommendations on the optimal timing of HSCT for aCML. However, the finding of increasing blast counts despite ruxolitinib treatment in this case might justify early intervention with allogeneic HSCT if a donor is available.

In conclusion, ruxolitinib for aCML with CSF3R muta- 
tion might not always induce a significant response but could be used as bridge to HSCT. The clinician should make an effort in searching for all eligible candidates for HSCT from the time of diagnosis of aCML. Prospective, collaborative studies incorporating new target agents are needed to provide the management guidelines of aCML, especially in pediatric cases.

\section{Conflict of Interest Statement}

The authors have no conflict of interest to declare.

\section{References}

1. Schwartz LC, Mascarenhas J. Current and evolving understanding of atypical chronic myeloid leukemia. Blood Rev 2019;33:74-81.

2. Gotlib J. How I treat atypical chronic myeloid leukemia. Blood 2017;129:838-45.

3. Dao KT, Tyner JW, Gotlib J. Recent progress in chronic neutrophilic leukemia and atypical chronic myeloid leukemia. Curr Hematol Malig Rep 2017;12:432-41.

4. Arber DA, Orazi A, Hasserjian R, et al. The 2016 revision to the World Health Organization classification of myeloid neoplasms and acute leukemia. Blood 2016;127:2391-405.

5. Dao KT, Gotlib J, Deininger MMN, et al. Efficacy of ruxolitinib in patients with chronic neutrophilic leukemia and atypical chronic myeloid leukemia. J Clin Oncol 2020;38: 1006-18.
6. Crisà E, Nicolosi M, Ferri V, Favini C, Gaidano G, Patriarca A. Atypical chronic myeloid leukemia: where are we now? Int J Mol Sci 2020;21:6862.

7. Freedman JL, Desai AV, Bailey LC, et al. Atypical chronic myeloid leukemia in two pediatric patients. Pediatr Blood Cancer 2016;63:156-9.

8. Maxson JE, Gotlib J, Pollyea DA, et al. Oncogenic CSF3R mutations in chronic neutrophilic leukemia and atypical CML. N Engl J Med 2013;368:1781-90.

9. Wang SA, Hasserjian RP, Fox PS, et al. Atypical chronic myeloid leukemia is clinically distinct from unclassifiable myelodysplastic/myeloproliferative neoplasms. Blood 2014;123:264551.

10. Breccia M, Biondo F, Latagliata R, Carmosino I, Mandelli F, Alimena G. Identification of risk factors in atypical chronic myeloid leukemia. Haematologica 2006;91:1566-8.

11. Dhakal P, Gundabolu K, Amador C, Rayamajhi S, Bhatt VR. Atypical chronic myeloid leukemia: a rare entity with management challenges. Future Oncol 2018;14:177-85.

12. Dao KH, Solti MB, Maxson JE, et al. Significant clinical response to JAK1/2 inhibition in a patient with CSF3R-T618Ipositive atypical chronic myeloid leukemia. Leuk Res Rep 2014:3:67-9.

13. Loh ML, Tasian SK, Rabin KR, et al. A phase 1 dosing study of ruxolitinib in children with relapsed or refractory solid tumors, leukemias, or myeloproliferative neoplasms: A Children's Oncology Group phase 1 consortium study (ADVL1011). Pediatr Blood Cancer 2015;62:1717-24.

14. Onida F, de Wreede LC, van Biezen A, et al. Allogeneic stem cell transplantation in patients with atypical chronic myeloid leukaemia: a retrospective study from the Chronic Malignancies Working Party of the European Society for Blood and Marrow Transplantation. Br J Haematol 2017;177:759-65. 